University of Texas at El Paso

ScholarWorks@UTEP

\title{
Reconstructing an Open Order from Its Closure, with Applications to Space-Time Physics and to Logic
}

Francisco Zapata

The University of Texas at El Paso

Vladik Kreinovich

The University of Texas at El Paso, vladik@utep.edu

Follow this and additional works at: https://scholarworks.utep.edu/cs_techrep

Part of the Computer Engineering Commons

Comments:

Technical Report: UTEP-CS-11-16b

To appear in Studia Logica, 2012, Vol. 100, No. 1-2.

\section{Recommended Citation}

Zapata, Francisco and Kreinovich, Vladik, "Reconstructing an Open Order from Its Closure, with Applications to Space-Time Physics and to Logic" (2011). Departmental Technical Reports (CS). 605. https://scholarworks.utep.edu/cs_techrep/605

This Article is brought to you for free and open access by the Computer Science at ScholarWorks@UTEP. It has been accepted for inclusion in Departmental Technical Reports (CS) by an authorized administrator of ScholarWorks@UTEP. For more information, please contact Iweber@utep.edu. 


\title{
F. ZAPATA Reconstructing an Open Order \\ V. Kreinovich from Its Closure, with \\ Applications to Space-Time Physics and to Logic
}

\begin{abstract}
In his logical papers, Leo Esakia studied corresponding ordered topological spaces and order-preserving mappings. Similar spaces and mappings appear in many other application areas such the analysis of causality in space-time. It is known that under reasonable conditions, both the topology and the original order relation $\preccurlyeq$ can be uniquely reconstructed if we know the "interior" $\prec$ of the order relation. It is also known that in some cases, we can uniquely reconstruct $\prec$ (and hence, topology) from $\preccurlyeq$. In this paper, we show that, in general, under reasonable conditions, the open order $\prec$ (and hence, the corresponding topology) can be uniquely determined from its closure $\preccurlyeq$.
\end{abstract}

Keywords: ordered topological space, order-preserving mappings, open and closed orders, space-time geometry, logic

\section{Formulation of the Problem}

Order-preserving mappings of topological spaces in logic and in physics: general reminder. Many interesting mathematical results appear when we are able to find connection between two seemingly different areas of mathematics - and thus, use known results and techniques from one area to study techniques from another area. In particular, for Heyting algebras - models of intuitionistic logics - many results originated from a relation between Heyting algebras and a special class of (partially) ordered topological spaces called Esakia spaces, a relation that was discovered and actively explored by Leo Esakia in [10, 11]. In his research, L. Esakia paid special attention to studying order-preserving maps between the corresponding partially ordered spaces.

Esakia's work was not the first application of ordered topological spaces and order-preserving mappings: such spaces and mappings also naturally appear in space-time physics and in other areas of logic.

In physics, a natural ordering relation is the causality relation between events, when $a \preccurlyeq b$ means that an event $a$ can influence the event $b$. 
In physical models of space-time, it is also assumed that there is a topology that corresponds to closeness of events. Intuitively, convergence $b_{n} \rightarrow b$ in this topology means that for any fixed measurement accuracy, when $n$ is sufficiently large, we cannot distinguish events $b_{n}$ and $b$ by measurements of this accuracy.

It is usually assumed that the relation $\preccurlyeq$ is "closed" in the sense that for every element $a$, its future cone $C_{a}^{+} \stackrel{\text { def }}{=}\{b: a \preccurlyeq b\}$ is closed. In other words, if a sequence $b_{n}$ converges to the element $b$ in the sense of the underlying topology, and $a \preccurlyeq b_{n}$ for all $n$, then $a \preccurlyeq b$.

This closeness makes intuitive sense. Indeed, if $b_{n} \rightarrow b$, this means, as we have mentioned, that for every given measurement accuracy, when $n$ is sufficiently large, we cannot distinguish events $b_{n}$ and $b$. So,

- if $a \preccurlyeq b_{n}$, i.e., if physical evidence shows $a$ can influence all events $b_{n}$, and

- $b_{n} \rightarrow b$, meaning that $b$ is indistinguishable from $b_{n}$,

then this same evidence shows that $a$ can influence $b(a \preccurlyeq b)$.

In Newton's physics, in which signals can potentially travel with an arbitrarily large speed (including immediate-action-at-a-distance, corresponding to infinite speed), the causality relation is trivial: an event $a=(t, x)$ occurring at the spatial location $x$ at time $t$ can influence the event $a^{\prime}=\left(t^{\prime}, x^{\prime}\right)$ if and only if $t \leq t^{\prime}$.

The fundamental role of the non-trivial causality relation emerged with the Special Relativity, according to which the speed of all the signals is limited by the speed of light $c$, as a result of which $a=(t, x) \preccurlyeq a^{\prime}=\left(t^{\prime}, x^{\prime}\right)$ if and only if $t^{\prime} \geq t$ and $\frac{d\left(x, x^{\prime}\right)}{t^{\prime}-t} \leq c$; see, e.g., [12].

Comment. For readers familiar withe the physics of special relativity, it should be mentioned that $a \preccurlyeq b$ means that $b$ is either inside or on the future light-cone based at $a$.

In the original relativity theory, causality was one of the main concepts. Its central role was revealed in the $1950 \mathrm{~s}$, when A. D. Alexandrov proved that in special relativity, causality implied Lorenz group: every order-preserving transforming of the corresponding partial ordered set is linear, and can be represented as a composition of spatial rotations, Lorentz transformations (describing a transition to a moving reference frame), and re-scaling $x \rightarrow \lambda \cdot x$ (corresponding to a change of unit for measuring space and time) $[1,5]$. This 
theorem was later generalized by E. Zeeman (and by many others) and is currently known as the Alexandrov-Zeeman theorem; see, e.g., [2, 3, 4, 6, 7, $13,14,15,16,17,18,21,23,25,27,28,29,30,31,32,36,40,45]$.

Special relativity theory is an approximate description of space-time, a description that does not take into account that space-time is curved. To describe curved space-times, we need General Relativity Theory and its generalizations. The notion of causality is the basis of several formalizations of space-time physics, both as foundations of the General Relativity Theory and as a way to describe its generalizations; see, e.g., [8, 26, 24, 25, 27, 41].

In logic, partial orders are used when we formalize commonsense and expert reasoning. In this application, to each statement, we assign the expert's degree of certainty that this statement is true. A natural partial ordering relation $a \preccurlyeq b$ describes the fact that we are more certain in $b$ than in $a$; see, e.g., $[20,34,38,39,43,44]$.

In this application, topology represents the closeness of the corresponding degrees of certainty. In this case, it is also reasonable to require that the relation $\preccurlyeq$ is closed.

Another important application of partial orders is decision making, when we need to describe human preferences [9, 22, 33, 37, 42].

Need for open partial orders. In many applications, we only observe an event $b$ with some accuracy. For example, in physics, we may want to check what is happening exactly 1 second after a certain reaction. However, in practice, we cannot measure time exactly, so, we can only observe an event which is close to $b-$ e.g., an event that occurs $1 \pm 0.001 \mathrm{sec}$ after the reaction. In general, we can only guarantee that the observed event is within a certain neighborhood $U_{b}$ of the event $b$.

Because of this uncertainty, the only possibility to experimentally confirm that $a$ can influence $b$ is when for some neighborhood $U_{b}$ of the event $b$, we have $a \preccurlyeq \widetilde{b}$ for all $\widetilde{b} \in U_{b}$. In topological terms, this "experimentally confirmable" relation $a \prec b$ means that $b$ is contained in the future cone $C_{a}^{+}=\{c: a \preccurlyeq c\}$ of the event $a$ together with some neighborhood, i.e., that $b$ belongs to the interior $K_{a}^{+}$of the closed cone $C_{a}^{+}$. Such relation, in which future cones are open, will be called open.

In usual space-time models, once we know the open cone $K_{a}^{+}$, we can reconstruct the original cone $C_{a}^{+}$as the closure of $K_{a}^{+}: C_{a}^{+}=\overline{K_{a}^{+}}$.

Comment. To avoid confusion, please note that here $a \prec b$ does not mean $a \preccurlyeq b$ and $a \neq b$. 
Similar arguments justify the need to consider open cones also in case of uncertainty.

In physics, there is another motivation for open cones: open cones correspond to influences with speeds smaller than the speed of light. This is important because, according to modern physics, there are two types of objects (see, e.g., [12]):

- objects with non-zero rest mass that can travel with any possible speed which is smaller than the speed of light - but not with the speed of light, and

- objects with zero rest mass (like photons), that can travel only with the speed of light, but not with any smaller speed.

Thus, open cones correspond to causality by traditional (kinematic) objects. Because of this, the open relation $a \prec b$ is also known as kinematic causality, and spaces with this open relation $\prec$ are known as kinematic spaces [41].

Comment. For example, in special relativity, $a \prec b$ means that $b-a$ is a future-oriented timelike vector.

Natural questions: what is the relation between open and closed partial orders? In all the above applications, on the same space, we have three things:

- topology;

- the original (closed) partial order $\preccurlyeq$; and

- the (open) partial order $\prec$.

It is reasonable to ask to what extent knowing only some of these things enables us to reconstruct the others.

Relation between open and closed partial orders: what is known. It is known that under some physically (and logically) reasonable assumptions, the open relation uniquely determines both the topology and the closed relation.

The corresponding topology was first introduced by A. D. Alexandrov and is thus known as Alexandrov topology. It is a topology whose base are open intervals $(a, b) \stackrel{\text { def }}{=}\{c: a \prec c \prec b\}$. For this definition to be valid, we need to make sure that intervals do form a base of a topology, i.e., when a 
point $x$ belongs to the intersection of two open intervals, there a whole open interval containing $x$ is contained in this intersection.

Once this topology is defined, we can define $a \preccurlyeq b$ as $b$ belonging to the closure $\overline{K_{a}^{+}}$of the open cone $K_{a}^{+}=\{c: a \prec c\}$. Of course, we need to make sure that a dual definition $a \in \overline{K_{b}^{-}}$, where $K_{b}^{-}=\{c: c \prec b\}$ leads to the exact same ordering.

It is also usually assumed that for every element $a$, there are elements larger than $a$ and smaller than $a$, and that if $a \prec b$, then there is a point in between $a$ and $b$.

Under these conditions, the above description determines the topology and the closed order in terms of the open order $\prec$. Thus, the open order uniquely determines both the topology and the closed order.

In the case of special relativity, the inverse is also true: if we know the closed partial order, then we can uniquely reconstruct the open order as well - and so, the topology. Hence, every 1-1 transformation preserving a closed order also preserves the open order and the topology. This conclusion is used in many proofs that every order-preserving transformation is linear. The proof of this conclusion is based on the easy-to-check observation that when $a \preccurlyeq b$, we have $a \prec b$ if and only if the relation $\preccurlyeq$ restricted to the closed interval $[a, b]=\{c: a \preccurlyeq c \preccurlyeq b\}$ is not a total (linear) order, i.e., if and only if there exist $c$ and $c^{\prime}$ for which $a \preccurlyeq c \preccurlyeq b, a \preccurlyeq c^{\prime} \preccurlyeq b, c \npreceq c^{\prime}$, and $c^{\prime} \npreceq c$.

It is known, however, that this observation does not hold in general. For example, on the 3 -D space $\mathbb{R}^{3}$ with a standard topology, we can define a component-wise partial order as follows: $a=\left(a_{1}, a_{2}, a_{3}\right) \preccurlyeq b=\left(b_{1}, b_{2}, b_{3}\right)$ if and only if $a_{1} \leq b_{1}, a_{2} \leq b_{2}$, and $a_{3} \leq b_{3}$. In this space, the corresponding open order is also easy to describe: $a=\left(a_{1}, a_{2}, a_{3}\right) \prec b=\left(b_{1}, b_{2}, b_{3}\right)$ if and only if $a_{1}<b_{1}, a_{2}<b_{2}$, and $a_{3} \leq b_{3}$. Here, however, we can have $a=(0,0,0) \preccurlyeq b=(0,1,1), a \nprec b$, but for $c=(0,0,1)$ and $c^{\prime}=(0,1,0)$, we have $a \preccurlyeq c \preccurlyeq b, a \preccurlyeq c^{\prime} \preccurlyeq b, c \npreceq c^{\prime}$, and $c^{\prime} \npreceq c$.

Remaining problem - that we solve in this paper. A natural question is: what happens in the general case? Can we uniquely reconstruct an open order if we know the corresponding closed order? In this paper, we show that under reasonable assumptions, such a reconstruction is indeed possible.

This work was motivated by our discussions with Leo Esakia during his visit to Las Cruces, New Mexico. 


\section{Definitions and the Main Result}

Definition 1. [41] A set $X$ with a partial order $\prec$ is called a kinematic space if is satisfies the following conditions:

$$
\begin{gathered}
\forall a \exists a_{-}, a_{+}\left(a_{-} \prec a \prec a_{+}\right) ; \\
\forall a, b(a \prec b \rightarrow \exists c(a \prec c \prec b)) ; \\
\forall a, b, c(a \prec b, c \rightarrow \exists d(a \prec d \prec b, c)) ; \\
\forall a, b, c(b, c \prec a \rightarrow \exists d(b, c \prec d \prec a)) .
\end{gathered}
$$

Definition 2. For every partial ordered set, and every $a \prec b$, by an interval (or open interval), we mean the set $(a, b) \stackrel{\text { def }}{=}\{c: a \prec c \prec b\}$.

Definition 3. A kinematic space is called separable if there exists a countable set $\left\{x_{n}\right\}$ such that every open interval contains one of the elements $x_{i}$.

Definition 4. [41] For every separable kinematic space, we define convergence $s_{n} \rightarrow a$ as follows:

$$
\left.s_{n} \rightarrow a \Leftrightarrow \forall a_{-}, a_{+}\left(a_{-} \prec a \prec a_{+} \Rightarrow \exists N \forall n\left(n \geq N \Rightarrow a_{-} \prec s_{n} \prec a_{+}\right)\right)\right) .
$$

For each set $S$, its closure $\bar{S}$ is defined as the set of all the points a for which $s_{n} \rightarrow$ a for some $\left\{s_{n}\right\} \subseteq S$.

Comment. In other words, $s_{n} \rightarrow a$ if and only if every interval $\left(a_{-}, a_{+}\right)$ containing $a$ also contains almost all elements of the sequence $s_{n}$ - i.e., in other words, that it contains all but finitely many of these elements.

Comment. From the physical viewpoint, the fact that we consider separable spaces is not really restrictive, because all space-time models considered in mainstream physics are separable:

- The original Minkowski space is separable.

- Modern physics describes space-time as a manifold equipped with pseudo-Riemannian metric. All such manifolds are separable. To describe singularities, physicists consider manifolds with borders; these spaces are also separable; see, e.g., a classical textbook [35]. 
Mathematical comment. In this paper, we consider separable kinematic spaces, i.e., spaces in which there is a countable set $\left\{x_{n}\right\}$ which is everywhere dense in $X$. In such spaces, to describe topology, it is sufficient to consider convergence of sequences. Our result, however, can be easily extended to general (not necessarily separable) kinematic spaces if, instead of sequences $\left\{s_{n}\right\}$, we consider nets $\left\{s_{\alpha}\right\}_{\alpha \in A}$ corresponding to directed sets $A$; see, e.g., [19].

Definition 5. [41] A kinematic space is called normal if

$$
b \in \overline{\{c: c \succ a\}} \Leftrightarrow a \in \overline{\{c: c \prec b\}} .
$$

Notation. For a normal kinematic space, we denote $b \in \overline{\{c: c \succ a\}}$ by $a \preccurlyeq b$. For every $a \preccurlyeq b$, the set $[a, b] \stackrel{\text { def }}{=}\{c: a \preccurlyeq c \preccurlyeq b\}$ is called a closed interval.

The following transitivity and closure properties hold for this relation:

Proposition 1. [41] For every separable normal kinematic space and for every elements $a, b$, and $c$, the following holds:

- $a \preccurlyeq a$;

- if $a \prec b$, then $a \preccurlyeq b$;

- if $a \preccurlyeq b$ and $b \prec c$, then $a \prec c$;

- if $a \prec b$ and $b \preccurlyeq c$, then $a \prec c$.

The proof of the first part of Proposition 1 is based on the following lemma:

Definition 6. We say that a sequence $\left\{s_{n}\right\}$ is $\prec$-decreasing if $s_{n} \succ s_{n+1}$ for all $n$.

Lemma 1. For every separable kinematic space, if $a \prec b$, then there exists $a \prec$-decreasing sequence $\left\{s_{n}\right\}$ of elements $s_{n} \succ a$ for which $s_{1}=b$ and $s_{n} \rightarrow a$.

Comment. For readers' convenience, all the proofs are placed in the special (final) Proofs section. 
A dual lemma also holds:

Definition 7. We say that a sequence $\left\{s_{n}\right\}$ is $\prec$-increasing if $s_{n} \prec s_{n+1}$ for all $n$.

Lemma 2. For every separable kinematic space, for every element a, there exists an $\prec$-increasing sequence $s_{n}$ of element $s_{n} \prec$ a for which $s_{n} \rightarrow a$.

Proposition 2. [41] For every separable normal kinematic space:

- if $s_{n} \succcurlyeq b$ for all $n$ and $s_{n} \rightarrow a$, then $a \succcurlyeq b$;

- if $s_{n} \preccurlyeq b$ for all $n$ and $s_{n} \rightarrow a$, then $a \preccurlyeq b$;

- if $a \preccurlyeq b$ and $b \preccurlyeq c$, then $a \preccurlyeq c$.

Comment. In the causality relations corresponding to Einstein's space-time models, the causality relation is an order relation. However, from the mathematical viewpoint, it is possible to define kinematic spaces in which the limit relation $\preccurlyeq$ is not an order, i.e., in which, for some elements $a$ and $b$, we have $a \preccurlyeq b$ and $b \preccurlyeq a$, but $a \neq b$.

For example, in Newtonian space-time, $e=(t, x) \preccurlyeq e^{\prime}=\left(t^{\prime}, x^{\prime}\right) \Leftrightarrow t \leq t^{\prime}$. In this order, $(0,(0,0,0)) \preccurlyeq(0,(1,0,0))$ and $(0,(1,0,0)) \preccurlyeq(0,(0,0,0))$, but $(0,(0,0,0)) \neq(0,(1,0,0))$.

In such non-order cases, we have a non-trivial equivalence relation $a \equiv$ $b \Leftrightarrow(a \preccurlyeq b \& b \preccurlyeq a)$. For each element $a$, its equivalence class $\{b: b \equiv a\}$ is equal to $[a, a]$.

To formulate our result, we need to introduce an additional completeness property.

Definition 8. We say that a sequence $\left\{s_{n}\right\}$ is $\preccurlyeq$-decreasing if $s_{n} \succcurlyeq s_{n+1}$ for all $n$.

Definition 9. We say that a sequence $\left\{s_{n}\right\}$ is bounded from below if there exists an element $b$ for which $b \preccurlyeq s_{n}$ for all $n$.

Definition 10. We say that a separable kinematic space is complete if every $\preccurlyeq$-decreasing bounded sequence has a limit. 
Physical comment. From the application viewpoint, this requirement does not change much. Indeed, the events are only approximately known anyway, so explicitly adding a limit event $a=\lim s_{n}$ does not affect the physical picture: for all practical purposes, the limit $a$ is indistinguishable from $s_{n}$ for large $n$.

The following result shows that completeness holds in most physically interesting cases, for example, in the space-time corresponding to special relativity and in many models corresponding to General Relativity.

Definition 11. A kinematic space is called intervally compact if in this space, every closed interval is compact.

Proposition 3. Every intervally-compact separable normal kinematic space is complete.

Definition 12. For every element $e \in X$, let $S_{e}$ denote the set of all $\preccurlyeq-$ monotonically decreasing sequences $s=\left\{s_{n}\right\}$ for which $e \preccurlyeq s_{n}$ for all $n$ and $\bigcap_{n=1}^{\infty}\left[e, s_{n}\right]=[e, e]$. On this set of sequences, we can define a new pre-ordering $s \geq s^{\prime} \Leftrightarrow \forall n \exists m\left(s_{n} \succcurlyeq s_{m}^{\prime}\right)$.

Definition 13. We say that a sequence $s \in S_{e}$ is $S_{e}$-maximum if $s \geq s^{\prime}$ for every $s^{\prime} \in S_{e}$.

Comment. For every $e$, we may have several different $S_{e}$-maximum sequences.

The following result states that a complete (open) kinematic order can indeed be uniquely reconstructed from the corresponding closed order.

Theorem. For every complete separable normal kinematic space, $a \succ b$ if and only if there exists an element $e \succcurlyeq b$ and a sequence $\left\{s_{n}\right\}$ which is $S_{e}$-maximum and for which $s_{1}=a$.

Comment. This theorem describes $\prec$ in terms of $\preccurlyeq$. Thus, the relation $\prec$ is preserved by an arbitrary $\preccurlyeq$-isomorphism between kinematic spaces. In other words, we arrive at the following corollary. 
Corollary. If two complete separable normal kinematic orders $\prec$ and $\prec^{\prime}$ on the same set $X$ lead to the same closed order $\preccurlyeq=\preccurlyeq^{\prime}$, then $\prec=\prec^{\prime}$.

Physical meaning of this result. One of the main objectives of physics is to reduce observed phenomena to fundamental theories and explanations. Because of this activity, it is important to try our best to describe seemingly less fundamental properties in terms of more fundamental ones. For example, wherever an empirical law is discovered, like the Ohm law that describes the relation between current and voltage, physicists try to reduce this empirical law to more fundamental laws of nature.

Another example of such a reduction was given earlier: Lorentz transformations - describing coordinates transformations during a transition to a moving reference frame - can be explained as transformations that preserve causality. This description reduces such less fundamental properties as coordinates and coordinate transformations to causality - one of the most fundamental notions of physics; see, e.g., [12].

The above result can be viewed as another example of such a reduction. Indeed, as we have mentioned, from the physical viewpoint,

- the relation $\preccurlyeq$ is the causality relation, while

- the relation $a \prec b$ means that $a$ can influence $b$ by using particle with positive rest mass - i.e., particles that travel with speeds smaller than the speed of light.

In physics:

- causality is one of the most fundamental properties, while

- the properties like the rest mass are less fundamental.

Thus, the fact that we can express $\prec$ in terms of $\preccurlyeq$ means that we are reducing the less fundamental relation - of being influences by particles of non-zero rest mass - to causality, one of the most fundamental notions of physics.

\section{Proofs}

\subsection{Proof of Lemma 1}

Since the kinematic space is separable, there exists a sequence $x_{n}$ that has elements in every open interval. We will construct a sequence $s_{n}$ with the following additional property: for every $n \geq 2$, if $x_{n} \succ a$, then $x_{n} \succ s_{n}$. 
Let us take $s_{1}=b$; then, $b \succ a$ implies that $s_{1} \succ a$.

Let us now assume that the values $s_{1} \succ \ldots \succ s_{n-1} \succ a$ have already been constructed. The construction of the next element $s_{n}$ will depend on whether $x_{n} \succ a$ or not. If $x_{n} \succ a$, then we have $a \prec x_{n}, s_{n-1}$. So, by definition of a kinematic space, there exists an element $c$ for which $a \prec c \prec x_{n}, s_{n-1}$. We will take one of these elements $c$ as $s_{n}$.

If $x_{n} \nsucc a$, then we have $a \succ s_{n-1}$. So, by definition of a kinematic space, there exists an element $c$ for which $a \prec c \prec s_{n-1}$. We will take one of these elements $c$ as $s_{n}$.

We have constructed a $\prec$-decreasing sequence. Let us prove that this sequence converges to $a$, i.e., that for every $a_{-} \prec a \prec a_{+}$, there exists an $N$ such that for all $n \geq N$, we have $a_{-} \prec a \prec a_{+}$. Indeed, since $a \prec a_{+}$, the sequence $x_{n}$ has an element $x_{N}$ in an open interval $\left(a, a_{+}\right): a \prec x_{N} \prec a_{+}$. By our construction, $x_{N} \succ a$ implies that $a \prec a_{N} \prec x_{N}$. By transitivity, we conclude that $a_{-} \prec a_{N} \prec a_{+}$. Since the sequence $s_{n}$ is $\prec$-decreasing, we conclude that for $n>N$, we have $a \prec s_{n} \prec s_{N}$, so, by transitivity, $a_{-} \prec s_{n} \prec a_{+}$. Convergence is proven.

\subsection{Proof of Lemma 2}

This proof is similar to the proof of Lemma 1.

\subsection{Proof of Proposition 1}

Let us first prove that $a \succcurlyeq a$. Indeed, by Lemma 1, there exists a sequence $s_{n}$ for which $s_{n} \succ a$ and $s_{n} \rightarrow a$. Thus, $a \succcurlyeq a$.

Let us prove that if $a \prec b$, then $a \preccurlyeq b$. Indeed, we can take $s_{n}=b$. Each open interval neighborhood of $b$ contains $b$ and thus, contains all elements of the sequence $s_{n}$. Thus, $s_{n} \rightarrow b$ and hence, $a \preccurlyeq b$.

Let us now prove that if $a \preccurlyeq b$ and $b \prec c$, then $a \prec c$. Indeed, by definition, $a \preccurlyeq b$ means that there is a sequence $s_{n} \rightarrow b$ for which $a \prec s_{n}$ for all $n$. By definition of convergence, $s_{n} \rightarrow b$ means that for every two elements $b_{-} \prec b \prec b_{+}$, there exists $N$ for which, for all $n \geq N, b_{-} \prec s_{n} \prec b_{+}$. By definition of a kinematic space, there is an element $b_{-} \prec b$. As $b_{+}$, we take $b_{+}=c$. In this case, for sufficiently large $n$, we have $s_{n} \prec c$, so $a \prec s_{n}$ and transitivity imply that $a \prec c$.

Finally, let us prove that if $a \prec b$ and $b \preccurlyeq c$, then $a \prec c$. Indeed, since the kinematic space is normal, $b \preccurlyeq c$ means that there exists a sequence $s_{n} \rightarrow b$ for which $s_{n} \prec c$ for all $n$. By definition of convergence, $s_{n} \rightarrow b$ means that for every two elements $b_{-} \prec b \prec b_{+}$, there exists $N$ for which, 
for all $n \geq N, b_{-} \prec s_{n} \prec b_{+}$. By definition of a kinematic space, there is an element $b_{+} \succ b$. As $b_{-}$, we take $b_{-}=a$. In this case, for sufficiently large $n$, we have $s_{n} \succ a$, so $s_{n} \prec c$ and transitivity imply that $a \prec c$.

Finally, let us prove that the relation $\succcurlyeq$ is transitive. Let $a \succcurlyeq b$ and $b \succcurlyeq c$. By definition, $a \succcurlyeq b$ means that there exists a sequence $s_{n} \succ b$ for which $s_{n} \rightarrow a$. As we have shown, from $s_{n} \succ b$ and $b \succcurlyeq c$, we conclude that $s_{n} \succ c$. Thus, $s_{n} \succ c$ for some sequence $s_{n} \rightarrow a$. This is exactly what is means to have $a \succcurlyeq c$. The statement is proven.

The proposition is proven.

\subsection{Proof of Proposition 2}

Without losing generality, let us prove the first statement, i.e., let us assume that $s_{n} \rightarrow a$ and $s_{n} \succcurlyeq b$, and let us prove that $a \succcurlyeq b$. For that, we will need to prove that there exists a sequence $s_{n}^{\prime} \succ b$ for which $s_{n}^{\prime} \rightarrow a$. As such a sequence, we will take a $\prec$-decreasing sequence $s_{n}^{\prime}$ for which $s_{n}^{\prime} \succ a$ and $s_{n}^{\prime} \rightarrow a$, a sequence whose existence was proved in Lemma 1 . Since $s_{n}^{\prime} \rightarrow a$, to complete our proof, it is sufficient to prove that $s_{n}^{\prime} \succ b$ for all $n$.

Indeed, let $n$ be an arbitrary natural number. By definition of a kinematic space, there exists an element $a_{-} \prec a$, so we have $a_{-} \prec a \prec s_{n}^{\prime}$. Since the element $a$ is contained in the open interval $\left(a_{-}, s_{n}^{\prime}\right)$ and $s_{n} \rightarrow a$, by definition of convergence, there exists an $N$ for which $a_{-} \prec a_{N} \prec s_{n}^{\prime}$. By definition, $s_{N} \geq b$ means that there exists a sequence of elements $s_{N, 1}, s_{N, 2}, \ldots$ for which $s_{N, k}>b$ and $s_{N, k} \rightarrow s_{N}$. Since $s_{N} \in\left(a_{-}, a_{n}^{\prime}\right)$, by definition of convergence, this implies that for some $K$, we have $s_{N, K} \in\left(a_{-}, s_{n}^{\prime}\right)$. From $s_{N, K} \prec s_{n}^{\prime}$ and $s_{N, K} \succ b$, we conclude that $s_{n}^{\prime} \succ b$. The proposition is proven.

\subsection{Proof of Proposition 3}

If $\left\{s_{n}\right\}$ is a $\leq$-decreasing bounded sequence, with a bound $b$, then all its elements belong to the interval $\left[b, s_{1}\right]$. Since the kinematic space is intervallycompact, this interval is compact. Thus, by known properties of compactness, the sequence $\left\{s_{n}\right\}$ has a convergent subsequence $s_{n_{k}} \rightarrow a$, where $n_{k} \rightarrow \infty$. By definition of the Alexandrov topology on a kinematic space, this means that for every $a_{-} \prec a \prec a_{+}$, there exists a $K$ for which, for all $k \geq K$, we have $a_{-} \prec s_{n_{k}} \prec a_{+}$. Let us show that $s_{n} \rightarrow a$, i.e., that for every $a_{-}$and $a_{+}$, there exists an $N$ for which, for all $n \geq N$, we have $a_{-} \prec s_{n} \prec a_{+}$. Indeed, let $K$ be the value corresponding to these $a_{-}$and $a_{+}$, and let us take $N=n_{K}$. In this case, $s_{N}=s_{n_{K}} \prec a_{+}$. 
When $n \geq N$, then, due to the fact that the sequence is $\preccurlyeq$-decreasing, we have $s_{n} \succcurlyeq s_{N}$, so due to $s_{N} \prec a_{+}$, we have $s_{n} \prec a_{+}$.

Since $n_{k} \rightarrow \infty$, there exists a value $k_{0} \geq K$ for which $n_{k_{0}} \geq n$ and hence, $s_{n_{k_{0}}} \preccurlyeq s_{n}$. Thus, from $a_{-} \prec s_{n_{k_{0}}}$ and $s_{n_{k_{0}}} \preccurlyeq s_{n}$, we conclude that $s_{-} \prec s_{n}$. Convergence is proven, and so it the proposition.

\subsection{Proof of the Theorem}

Our proof is based on the following three lemmas:

Lemma 3. For every complete separable normal kinematic space, if $s \in S_{e}$, then $s_{n} \rightarrow e$.

Lemma 4. For every complete separable normal kinematic space, if $s_{n} \rightarrow$ $e$, and $\left\{s_{n}\right\}$ is $\prec$-decreasing, then the sequence $\left\{s_{n}\right\}$ is in $S_{e}$.

Lemma 5. For every complete separable normal kinematic space, if a sequence $s \in S_{e}$ is $\prec$-decreasing, then it is $S_{e}$-maximum.

Proof of Lemma 3. If $s \in S_{e}$, then $s_{n}$ is a $\preccurlyeq$-decreasing sequence which is bounded by $e$. Since the kinematic space is complete, this sequence has a limit. Let us denote this limit by $b$.

From $s_{n} \succcurlyeq e$ and $s_{n} \rightarrow b$, in the limit, we get $b \succcurlyeq e$; see Proposition 2 . From the fact that $s_{N} \preccurlyeq s_{n}$ for all $N \geq n$, in the limit, we get $b \preccurlyeq s_{n}$ for all $n$. Thus, $e \preccurlyeq b \preccurlyeq s_{n}$ for all $n$, i.e., $b$ belongs to all the closed intervals $\left[e, s_{n}\right]$ and so, $b$ belongs to the intersection $[e, e]$ of all these closed intervals.

The fact that $b \in[e, e]$ means that $b \preccurlyeq e$ and $e \preccurlyeq b$. Now, for every element $x$, if $x \prec b$ then from $x \prec b$ and $b \prec e$, we conclude that $x \prec e$. Vice versa, if $x \prec e$, then from $x \prec e$ and $e \preccurlyeq b$, we conclude that $x \prec b$. Thus, $x \prec b$ if and only if $x \prec e$. Similarly, for every element $x$, we have $b \prec x$ if and only if $e \prec x$. So, in terms of the open relation $\prec$, the elements $e$ and $b$ are interchangeable. Since the limit is defined in terms of the open relation $\prec$, the fact that $s_{n} \rightarrow b$ implies that $s_{n} \rightarrow e$. The lemma is proven.

Proof of Lemma 4. By definition of the class $S_{e}$, to prove the lemma, we must prove that $e \preccurlyeq s_{n}$ for all $n$ and that $\bigcap_{n=1}^{\infty}\left[e, s_{n}\right]=[e, e]$.

Let us first prove that for every $n$, we have $e \preccurlyeq s_{n}$. Indeed, since the sequence $\left\{s_{n}\right\}$ is <-decreasing, for every $m>0$, we have $s_{n+m} \prec s_{n}$ and 
thus, $s_{n+m} \preccurlyeq s_{n}$. From $s_{n} \rightarrow e$, we conclude that $s_{n+m} \rightarrow e$. Due to Proposition $2, s_{n+m} \preccurlyeq s_{n}$ and $s_{n+m} \rightarrow e$ imply that $e \preccurlyeq s_{n}$.

Let us now prove that $\bigcap_{n=1}^{\infty}\left[e, s_{n}\right]=[e, e]$. For that, we prove that every element from the intersection belongs to the interval $[e, e]$, and that every element from the interval $[e, e]$ belongs to the intersection. Indeed, let $t$ be an element from the intersection $\bigcap_{n=1}^{\infty}\left[e, s_{n}\right]$. This means that for every $n$, $t \in\left[e, s_{n}\right]$, i.e., $e \preccurlyeq t$ and $t \preccurlyeq s_{n}$. Due to Proposition $2, t \preccurlyeq s_{n}$ and $s_{n} \rightarrow e$ imply that $t \preccurlyeq e$. Thus, $e \preccurlyeq t$ and $t \preccurlyeq e$, i.e., indeed, $t \in[e, e]$.

Vice versa, let $t \in[e, e]$, i.e., let $e \preccurlyeq t$ and $t \preccurlyeq e$. Let us prove that $t$ belongs to the intersection $\bigcap_{n=1}^{\infty}\left[e, s_{n}\right]$, i.e., that for every $n$, we have $t \in\left[e, s_{n}\right]$. Indeed, we know that $e \preccurlyeq t$. Due to Proposition 2, from $t \preccurlyeq e$ and $e \preccurlyeq s_{n}$, we conclude that $t \preccurlyeq s_{n}$. Thus, $e \preccurlyeq t \preccurlyeq s_{n}$, i.e., indeed, $t \in\left[e, s_{n}\right]$. The lemma is proven.

Proof of Lemma 5. Let us show that if $s$ is an $\prec$-decreasing element of $S_{e}$ and $s^{\prime} \in S_{e}$, then $s \geq s^{\prime}$, i.e., that for every $n$, there exist an $m$ for which $s_{n} \succcurlyeq s_{m}^{\prime}$. Indeed, by Lemma $3, s^{\prime} \in S_{e}$ implies that $s_{n}^{\prime} \rightarrow e$. By definition of convergence, this means that for every $a_{-} \prec e \prec a_{+}$, there exists an $m_{0}$ for which, for all $m \geq m_{0}$, we have $a_{-} \prec s_{m}^{\prime} \prec a_{+}$.

By definition of a kinematic space, there exists an element $a_{-} \prec e$. Since $s_{n} \succ s_{n-1}$ and $s_{n-1} \succcurlyeq e$, we conclude, by Proposition 2, that $s_{n} \succ e$. So, we can take $a_{+}=s_{n}$. Then, there exists an $m$ for which $s_{m}^{\prime} \prec a_{+}=s_{n}$ and thus, $s_{m}^{\prime} \preccurlyeq s_{n}$. The Lemma is proven.

Proof of the Theorem. Let $a \succ b$. Then, according to Lemma 1, we can construct a $\prec$-decreasing sequence $s_{n}$ for which $s_{1}=a, s_{n} \succ b$, and $s_{n} \rightarrow b$. Due to Lemma 4 , we can thus conclude that $s \in S_{b}$. So, due to Lemma 5 , we conclude that the sequence $s$ is $S_{b}$-maximum.

Vice versa, let us assume that for some $e \succcurlyeq b$, there exists an $S_{e^{-}}$ maximum sequence $s$ for which $a=s_{1}$. This means that for every other sequence $s^{\prime} \in S_{e}$, we have $s \geq s^{\prime}$. In particular, as $s^{\prime}$, we can take a $\prec-$ decreasing sequence $s_{n}^{\prime}$ for which $s_{n}^{\prime} \rightarrow e$. For this sequence, $s_{n}^{\prime} \succ e$ for all $n$. From $s \geq s^{\prime}$, we conclude, in particular, that there exists an $m$ for which $a=s_{1} \succcurlyeq s_{m}^{\prime}$. From $a \succcurlyeq s_{m}^{\prime} \succ e \succcurlyeq b$, we now conclude - via Proposition 1 that $a \succ b$.

The Theorem is proven. 
Acknowledgements. This work was partly supported by a CONACyT scholarship, by the National Science Foundation grants HRD-0734825 and DUE-0926721, and by Grant 1 T36 GM078000-01 from the National Institutes of Health.

The authors are thankful to all the participants of the 2006 Dagstuhl seminar on Computational Structures for Modeling Space, Time, and Causality, especially to Ralph Kopperman, Prakash Panangaden, Michael B. Smith, and Dieter Spreen for stimulating discussions, to Guram Bezhanishvili for the invitation to participate in this special issue, to Leo Esakia himself for insightful ideas, and to the anonymous referees for their thorough reading and valuable suggestions.

\section{References}

[1] Alexandrov, A. D., 'On Lorentz transformations', Uspekhi Math. Nauk 5(3): 187, 1950 (in Russian).

[2] Alexandrov, A. D., 'A contribution to chronogeometry', Canadian Journal of Mathematics, 19:1119-1128, 1967.

[3] Alexandrov, A. D., 'Mappings of spaces with families of cones and space-time transformations', Annali di Matematica Pura ed Aplicata, 103:229-257, 1967.

[4] Alexandrov, A. D., 'On the axioms of relativity theory', Vestnik Leningrad University, Ser. Math., 19:5-28, 1967.

[5] Alexandrov, A. D., and V. V. Ovchinnikova, 'Remarks on the foundations of special relativity', Leningrad University Vestnik, No. 11:94-110, 1953 (in Russian).

[6] Benz, W., 'A characterization of plane Lorentz transformations', Journal of Geometry, 10:45-56, 1977.

[7] Benz, W., Geometrische Transformationen, BI Wissenscahftsverlag, Mannheim, 1992.

[8] Busemann, H., Timelike spaces, PWN Publishers, Warszawa, 1967.

[9] de la Mora, C., P. Wojciechowski, V. Kreinovich, S. A. Starks, P. Tanenbaum, and A. Kuzminykh, 'Robust Methodology for Characterizing System Response to Damage: A Subjective (Fuzzy) Partial Ordered Modification of the Traditional Utility-Probability Scheme', Proceedings of the 22nd International Conference of the North American Fuzzy Information Processing Society NAFIPS'2003, Chicago, Illinois, July 24-26, 2003, pp. 413-419.

[10] Esakia, L., 'Topological Kripke models', Soviet Math Dokl. 15:147-151, 1974.

[11] Esakia, L., Heyting Algebras I. Duality Theory, Metsniereba, Tbilisi, 1985 (in Russian).

[12] Feynman, R. R. Leighton, and M. Sands, The Feynman Lectures on Physics, Addison Wesley, Boston, Massachusetts, 2005.

[13] Guts, A. K., 'On mappings of families of sets', Soviet Math, Dokl., 14:506-508, 1973.

[14] Guts, A. K., 'On mappings preserving cones in a Lobachevski space', Math. Notes, 13:411-415, 1973. 
[15] Guts, A. K., 'Mappings of an ordered Lobachevsky space', Soviet Math. Dok., 15:416419, 1974.

[16] Guts, A. K., 'Invariant orders on three-dimensional Lie groups', Siberian Math. Journal, 17:731-736, 1976

[17] Guts, A. K., 'The chronogeometry of Gödel and de Sitter manifolds', Siberian Math. Journal, pp. 509-513, 1980.

[18] Guts, A. K., 'Axiomatic relativity theory', Russian Math. Surveys, 37(2):41-89, 1982.

[19] Kelly, J. L., General Topology, Sprinegr Verlag, Berlin, Heidelberg, New York, 1995.

[20] Klir, G., and B. Yuan, Fuzzy Sets and Fuzzy Logic: Theory and Applications, Prentice Hall, Upper Saddle River, New Jersey, 1995.

[21] Kosheleva, O. M., V. Kreinovich, and P. G. Vroegindewey, 'An extension of a theorem of A. D. Alexandrov to a class of partially ordered fields', Proceedings of the Royal Academy of Science of Netherlands, Series A, 82(3):363-376, 1979.

[22] Kosheleva, O.M., V. Kreinovich, H. T. Nguyen, and B. Bouchon-Meunier, 'How to describe partially ordered preferences: mathematical foundations', In: Nguyen, H.P., and A. Ohsato (eds.), Proceedings of the Vietnam-Japan Bilateral Symposium on Fuzzy Systems and Applications VJFUZZY'98, HaLong Bay, Vietnam, 30th September-2nd October, 1998, pp. 269-278.

[23] Kreinovich, V., 'Approximately measured causality implies the Lorentz group: Alexandrov-Zeeman result made more realistic', International Journal of Theoretical Physics, 33(8):1733-1747, 1994.

[24] Kreinovich, V., 'Symmetry characterization of Pimenov's spacetime: a reformulation of causality axioms', International Journal of Theoretical Physics, 35(2):341-346, 1996.

[25] Kreinovich, V., and O. Kosheleva, 'Computational Complexity of Determining Which Statements about Causality Hold in Different Space-Time Models', Theoretical Computer Science, 405(1-2):50-63, 2008.

[26] Kronheimer, E.H., and R. Penrose, 'On the structure of causal spaces', Proc. Cambr. Phil. Soc., 63(2):481-501, 1967.

[27] Künzi, H.-P. A., and V. Kreinovich, 'Static Space-Times Naturally Lead to QuasiPseudometrics', Theoretical Computer Science, 405(1-2):64-72, 2008.

[28] Kuzminykh, A.V., 'Characterization of Lorentz transformations', Soviet Math. Dokl., 16(6):1626-1628, 1975.

[29] Kuzminykh, A. V., 'Minimal condition determining the Lorentz transformations', Siberian Mathematical Journal, 17(6):968-972, 1976.

[30] Lester, J. A., 'On null cone preserving mappings', Proceedings of Cambridge Mathematical Society, 81:455-462, 1977.

[31] Lester, J. A., 'Cone preserving mappings for quadratic cones over arbitrary fields', Canadian Journal of Mathematics, 29:1247-1253, 1977.

[32] Lester, J.A., 'A physical characterization of conformal transformations of Minkowski spacetime', Annals of Discrete Mathematics, 18:567-574, 1983.

[33] Luce, R. D., and R. RAIFA, Games and decisions: introduction and critical survey, Dover, New York, 1989.

[34] Mendel, J. M., Uncertain Rule-Based Fuzzy Logic Systems: Introduction and New Directions, Prentice-Hall, 2001. 
[35] Misner, C. W., K. S. Thorne, and J. A. Wheeler, Gravitation, Freeman, San Francisco, 1973.

[36] Naber, G. L., The geometry of Minkowski space-time, Springer-Verlag, N.Y., 1992.

[37] Nguyen, H. T., O. Kosheleva, and V. Kreinovich, 'Decision Making Beyond Arrow's Impossibility Theorem', International Journal of Intelligent Systems, 24(1):2747, 2009.

[38] Nguyen, H. T., V. Kreinovich, and Q. Zuo, 'Interval-valued degrees of belief: applications of interval com- putations to expert systems and intelligent control', International Journal of Uncertainty, Fuzziness, and Knowledge-Based Systems (IJUFKS), 5(3):317-358, 1997.

[39] Nguyen, H. T., and E. A. Walker, A First Course in Fuzzy Logic, Chapman \& Hall/CRC, Boca Raton, Florida, 2006.

[40] Pambuccian, V., 'Alexandrov-Zeeman type theorems expressed in terms of definability', Aequationes Mathematicae, 74:249-261, 2007.

[41] Pimenov, R. I., Kinematic spaces: Mathematical Theory of Space-Time, Consultants Bureau, New York, 1970.

[42] Tanenbaum, P.J., C.de la Mora, P. Wojciechowski, O. Kosheleva, V. Kreinovich, S. A. Starks, and A. Kuzminykh, 'Robust Methodology for Characterizing System Response to Damage: Approach Based on Partial Order', In: I. Lirkov, S. Margenov, J. Wasniewski, and P. Yalamov (eds.), Large-Scale Scientific Computing, Proceedings of the 4-th International Conference LSSC'2003, Sozopol, Bulgaria, June 4-8, 2003, Springer Lecture Notes in Computer Science, 2907:276-283, 2004.

[43] Zapata, F., O. Kosheleva, and K. Villaverde, 'Products of Partially Ordered Sets (Posets) and Intervals in Such Products, with Potential Applications to Uncertainty Logic and Space-Time Geometry', Abstracts of the 14th GAMM-IMACS International Symposium on Scientific Computing, Computer Arithmetic and Validated Numerics SCAN'2010, Lyon, France, September 27-30, 2010, pp. 142-144.

[44] Zapata, F., O. Kosheleva, and K. Villaverde, 'How to Tell When a Product of Two Partially Ordered Spaces Has a Certain Property: General Results with Application to Fuzzy Logic', Proceedings of the 30th Annual Conference of the North American Fuzzy Information Processing Society NAFIPS'2011, El Paso, Texas, March 18-20, 2011.

[45] Zeeman, E.C., 'Causality implies the Lorentz group", Journal of Mathematical Physics, 5:490-493, 1964.

FRANCISCO ZAPATA

Department of Computer Science

University of Texas at El Paso

$500 \mathrm{~W}$. University

El Paso, TX 79968, USA

fazg74@gmail.com 


\section{Vladik Kreinovich}

Department of Computer Science

University of Texas at El Paso

$500 \mathrm{~W}$. University

El Paso, TX 79968, USA

vladik@utep.edu 